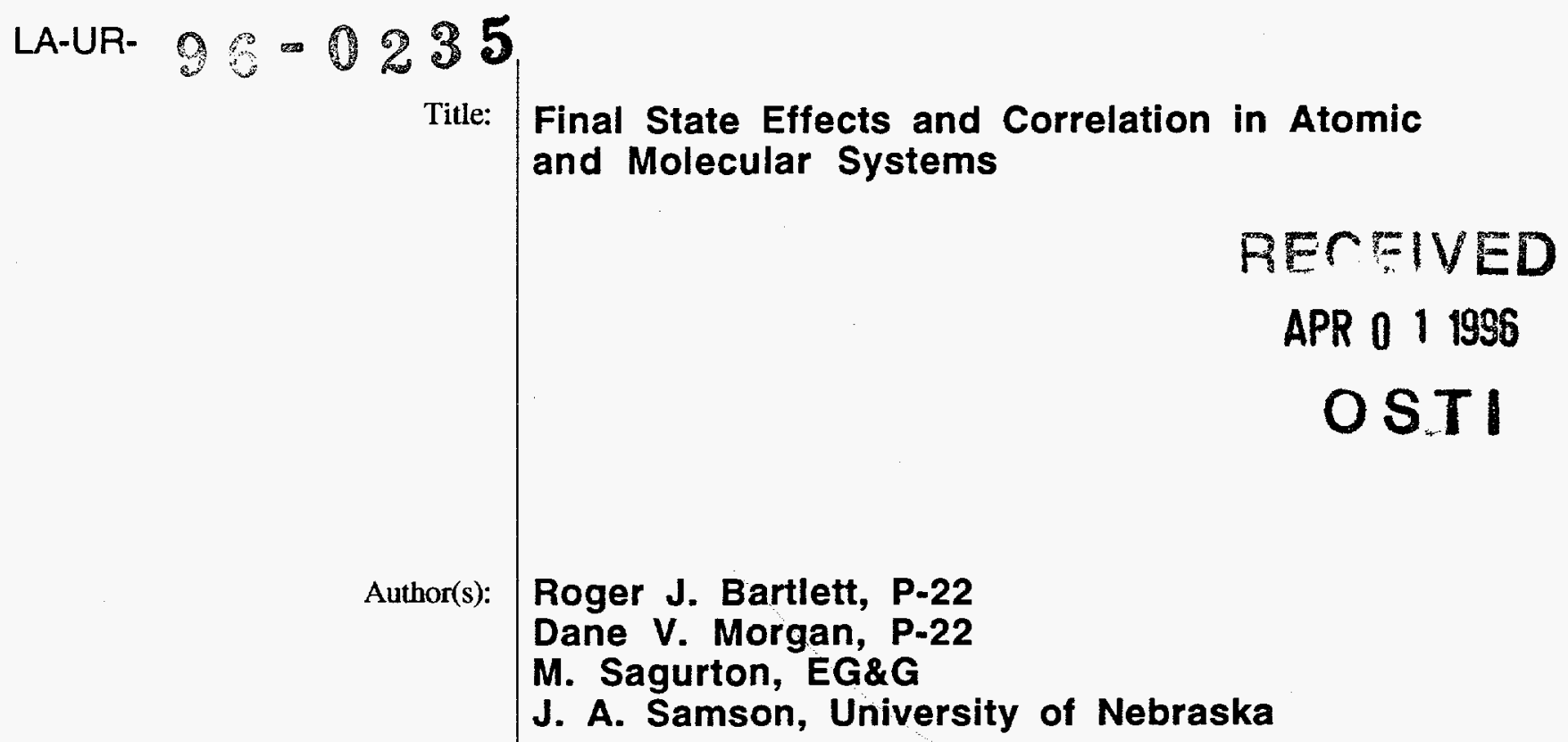

Submitted to:

DOE Office of Scientific and Technical Information (OSTI)

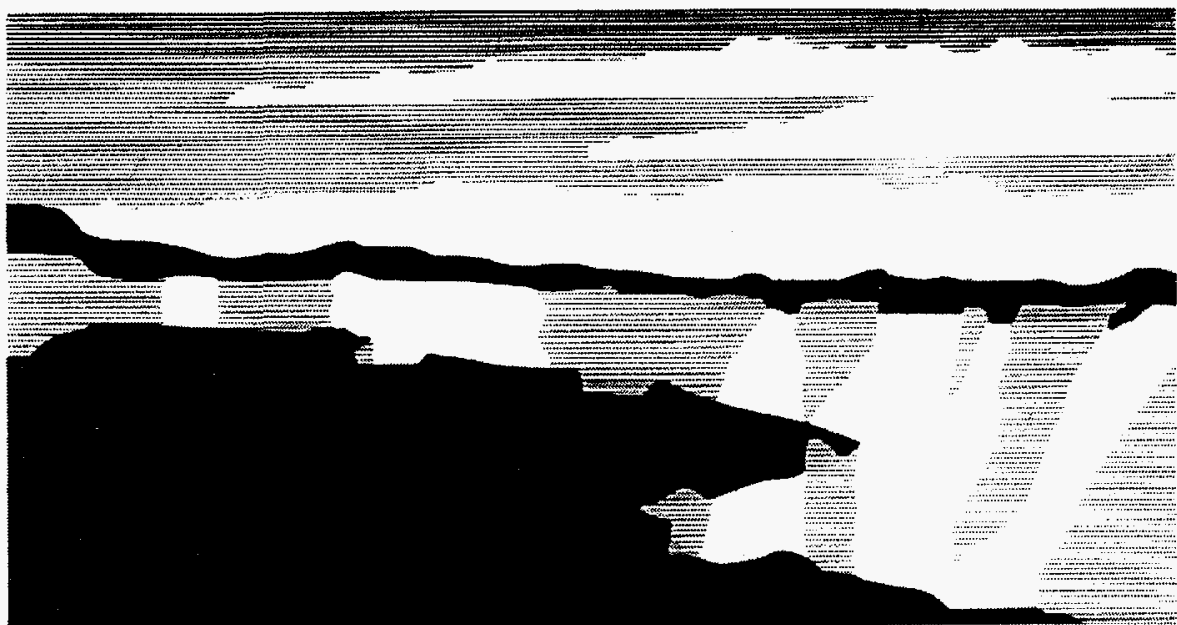

Los Alamos National Laboratony, an affirmative action/equal opportunity employer, is operated by the University of California for the U.S. Department of Energy under contract W-7405-ENG-36. By acceptance of this article, the publisher recognizes that the U.S. Government retains a nonexclusive, royattyfree license to publish or reproduce the published form of this contribution, or to allow others to do so, for U.S. Govemment purposes. The Los Alamos National Laboratory requests that the publisher Identity this article as work performed under the auspices of the U.S. Department of Energy.

$$
\text { DISTRIEUTION OF THB DOCUENT IS UHMTED }
$$




\title{
Final State Effects and Correlation in Atomic and Molecular Systems
}

Roger J. Bartlett*, Dane V. Morgan, M. Sagurton, and J. A. Samson (U. Nebraska)

\begin{abstract}
This is the final report of a three-year Laboratory-Directed Research and Development (LDRD) project at the Los Alamos National Laboratory (LANL). The project's goals were to experimentally examine phenomena caused by final state interactions of the photo-excited electron with the remaining ionic system. This includes multiple ionization by a single photon, which cannot be described solely within a one-electron framework, but requires explicit incorporation of correlation effects between the second electron and the primary electron. Having only two electrons, helium is one of the simplest systems which exhibits electron correlation and thus is an ideal choice for testing electron correlation models in atoms. We measured the double to single ionization ratio in helium and the multiple ionization ratio in several other rare gases to test the recent theories. We found an unexpected ionization channel caused by Compton scattering.
\end{abstract}

\section{Background and Research Objectives}

Recent advances in experimental techniques, particularly photon sources such as high brightness lasers and synchrotrons, have rekindled an interest in atomic and molecular physics. These research tools have made it possible to study in more detail than ever before the structure of atoms, molecules, and solids and the dynamics of their interaction with radiation. These studies have revealed many interesting phenomena that transcend the independent particle model and can be used to test more detailed theories and models. There have been parallel advances on the theoretical side enhanced by the development of large high-speed computers.

The interaction of electromagnetic radiation with matter is important in many pure everyday experiences. Phenomena as diverse as microwave heating, atmospheric science, photochemistry, weapons physics, astrophysics, energy production, the few-body coulomb

*Principal investigator, e-mail: rbartlett@lanl.gov 
problem, are all affected by photon-matter interactions. Most of these interactions are well understood and, with the development of supercomputers, detailed calculations have revealed and/or accounted for most of the observed phenomena. However, with the development of new photon sources such as lasers and synchrotrons, atomic, molecular, cluster and solid systems are being probed in such detail that even many of the most sophisticated theories are not adequate to explain the observed phenomena.

Cross sections for excitation, ionization, and dissociation are essential in many applied programs such as weapons physics or inertial confinement fusion (ICF), and it is important to determine the effects of such phenomena as Rydberg states, Compton scattering, multiple ionization and excitation and other final state effects on the cross sections. It is this latter area that is addressed in this project. These effects signal the breakdown of the independent particle model and are the cause of many interesting phenomena. The final state interactions affect the way radiation interacts with matter by opening final state channels that would otherwise be forbidden. This can have significant effects on cross sections and process rates influencing radiation transport, energy deposition and radiation damage.

Our overall goal was to experimentally examine phenomena caused by final state interactions of the photo-excited electron with the remaining ionic system. These events include multiple ionization by a single photon, ionization with excitation, and dissociation with other excitations in molecular and cluster systems. Specific objectives were to look at atoms, molecules and small clusters in order to broaden the types of systems studied and to determine the effects of the molecular/cluster interactions.

\section{Importance to LANL's Science and Technology Base and National R\&D Needs}

Multiple ionization and/or excitation processes are important because they epitomize the breakdown of the independent-particle model and they can provide important clues for the understanding of electron correlation and of excitation dynamics. Our studies are indicating where and when these processes cause significant deviation from the single particle-model results. Multiple ionization/excitation processes affect radiation transport, the rate of photon energy deposition, the time evolution of plasmas, etc. Thus they are important in fields as varied as nuclear weapons design to the effects of pollution on global climate change. They are also important in laser energy deposition thus playing a role in ICF.

It should be noted that a strong effort in programs that use synchrotron radiation will provide the drive for new photon sources such as ultraviolet free-electron lasers, laser produced plasmas sources and Smith-Purcell generated radiation. The Department of Energy 
(DOE) is expending significant resources on synchrotron radiation facilities. These include the National Synchrotron Light Source (NSLS) where we have our beam lines, the Advanced Photon Source at Argonne National Laboratory, the Advanced Light Source at Lawrence Berkeley Laboratory and the Stanford Synchrotron Radiation Laboratory. Our project has been one connection with this large scientific community.

\section{Scientific Approach and Results to Date}

We studied the effects of electron correlation and final state interactions in atomic, molecular and cluster systems as exemplified by multiple ionization and/or dissociation. The effects include but are not limited to autoionization, post-collision interactions, molecular and cluster dissociation and Auger decay. Photons provide an excellent probe to study these systems.

We used synchrotron radiation to provide a tunable, intense, polarized, high-resolution photon source to probe atomic, molecular and cluster systems and a time-of-flight spectrometer to measure the production and charge state of the ions. An electron energy analyzer was added to the ion-state spectrometer to allow coincidence measurements between an energy-selected electron and an ion-charge state.

More specifically the following tasks have been addressed:

\section{a) Double Ionization of Helium}

Measurement of the double and single photoionization cross sections of $\mathrm{He}$ from threshold to the high hv limit is of interest because of its relationship to the problem of describing electron correlation. In recent theoretical studies [1], many-body perturbation theory (MBPT) has been used to calculate the correlated two-electron initial- and final-state wave functions associated with photoabsorption-induced single and double ionization in $\mathrm{He}$ (the simplest neutral atom to exhibit correlation). Questions involve the number of terms which must be retained in the perturbation expansion as a function of hv and the accuracy with which individual terms can be evaluated. The test of theory has been the prediction of the hvdependent ratio of the double to single photoionization cross sections, $\sigma^{++} / \sigma^{+}[2]$. However, we have recently emphasized that for hv $>\sim 2.5 \mathrm{keV}$ Compton scattering begins to contribute significantly to the ionization cross sections, and in fact dominates $\sigma^{++} / \sigma^{+}$for hv above $\sim 8$ $\mathrm{keV}$ [3]. Experiments to date have measured only the total $\sigma^{++} / \sigma^{+}$without discriminating between the contributions from photoabsorption and Compton scattering.

These developments have motivated efforts to measure separately the ionization cross sections for Compton scattering and photoabsorption, especially in the crossover regime $2<\mathrm{hv}<8 \mathrm{keV}$. Recently, we reported the first measurements of $\mathrm{He}+$ produced through 
Compton scattering only for $2.5<\mathrm{hv}<5 \mathrm{keV}$ [4]. We have also made time-of-flight (TOF) measurements in which $\mathrm{He}+$ ions produced through Compton scattering and photoabsorption are time resolved, enabling a determination of the ratio $\sigma_{\mathrm{c}}{ }^{+} / \sigma_{\mathrm{p}}{ }^{+}$of the corresponding single photoionization cross sections. Our method exploits the fact that following photoabsorption of an $\mathrm{x}$-ray photon, the $\mathrm{He}+$ ion recoils in a direction opposite that of the photoemitted electron, which in turn has a cos $2 q$ distribution about the axis of the electric field. In contrast, the ion has only a small recoil velocity following Compton ionization because the electron excitation is generally to just above threshold. Thus, by choosing the axis of the TOF spectrometer to be collinear with the electric field, one observes two photoabsorption peaks corresponding to ion recoil toward and away from the detector, and a central Compton peak. We have carried out measurements for hv between 2 and $5 \mathrm{keV}$ on beam line X8A at the NSLS. The three peaks are clearly resolved and the results demonstrate the expected rapid increase in $\sigma_{\mathrm{c}}{ }^{+} / \sigma_{\mathrm{p}}{ }^{+}$with increasing hu in this range.

\section{b) Neon and Argon Photoionization Cross Section Measurements in} the Soft X-ray Range

Neon and argon are technological useful gases and are often used as fill gases in proportional counters. Also, because of their relative simplicity they are used as model systems to test theoretical calculations. The K-edge of neon is $\sim 0.87 \mathrm{keV}$. This is a difficult photon energy range in which to work and thus very few high resolution spectra exist. However, the LANL soft $x$-ray line at the NSLS covers this energy range and was used for our measurements. We completed measuring the partial ionization cross sections for several ionization states of neon and argon from $\sim 0.85 \mathrm{keV}$ to $5.5 \mathrm{keV}$. These measurements also exemplify electron correlation but are more difficult to interpret because of the many more ionization channels available in the interaction. The argon data from these measurements have been analyzed and submitted for publication in Phys. Rev. A. The neon data are still being analyzed.

\section{c) Project Summary}

The one electron model has found wide spread use in many areas of physics because of its calculational simplicity. However, the model does not faithfully reproduce phenomena such as multiple ionization caused by electron-electron interactions. To determine the limits of the model and the strength of the electron-electron interactions, we studied phenomena that are caused by electron correlation and many-body processes. To test recent theories that incorporate the electron-electron interactions, we measured the double ionization of $\mathrm{He}$ at photon energies well above the ionization threshold and the multiple ionization of argon and neon near the K-edges in these materials using the Los Alamos soft $\mathrm{x}$-ray synchrotron 
radiation source and a time-of-flight ion-state spectrometer. During the last year of the project, four papers were written and submitted for publication on the following topics:

- Single-Photon Multiple Ionization of Argon in the K-Edge Region

- The Effect of Compton Scattering on the Double to Single Photoionization Ratio in Helium

- Measurement of the Double-to-Single Photoionization Ratio in Compton Scattering from Helium for hv $=12 \mathrm{keV}$

- Single-Photon Multiple Ionization of Neon in the K Edge Region

\section{References}

[1] K. Hino, T. Ishihara, F. Shimizu, N. Toshima, and J.H. McGuire, Phys. Rev. B48, 1271 (1991), and references therein.

[2] J.C. Levin, I.A. Sellin, B.M. Johnson, D.W.Lindle, R.D. Miller, N. Berrah Monsour, Y. Azuma, H.G. Berry, and D.-H. Lee, Phys. Rev. A47, R16 (1993).

[3] J.A.R. Samson, C.H.Greene, and R.J. Bartlett, Phys. Rev. Lett. 71, 201 (1993).

[4] J.A.R. Samson, Z.X. He, R. J. Bartlett, and M. Sagurton, Phys. Rev. Lett., 72, 3329 (1994).

\section{DISCLAIMER}

This report was prepared as an account of work sponsored by an agency of the United States Government. Neither the United States Government nor any agency thereof, nor any of their employees, makes any warranty, express or implied, or assumes any legal liability or responsibility for the accuracy, completeness, or usefulness of any information, apparatus, product, or process disclosed, or represents that its use would not infringe privately owned rights. Reference herein to any specific commercial product, process, or service by trade name, trademark, manufacturer, or otherwise does not necessarily constitute or imply its endorsement, recommendation, or favoring by the United States Government or any agency thereof. The views and opinions of authors expressed herein do not necessarily state or reflect those of the United States Government or any agency thereof. 